\title{
Ultradian Oscillators in Somite Segmentation and Other Biological Events
}

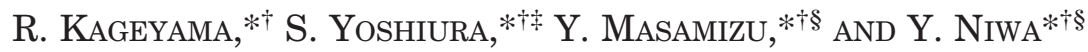 \\ *Institute for Virus Research, Kyoto University, Kyoto 606-8507, Japan; 'Japan Science and Technology Agency, \\ Core Research for Evolutional Science and Technology, Kyoto 606-8507, Japan; ${ }^{\circledR}$ Kyoto University Graduate School \\ of Biostudies, Kyoto 606-8502, Japan; ${ }^{\S}$ Kyoto University Graduate School of Medicine, Kyoto 606-8501, Japan
}

\begin{abstract}
Somite formation occurs every 2 hours in mouse embryos by periodic segmentation of the anterior ends of the presomitic mesoderm, and this process is controlled by a biological clock called the segmentation clock. During this process, the basic helix-loop-helix gene Hes 7 is cyclically expressed, and each cycle leads to generation of a bilateral pair of somites. Both sustained expression and loss of expression of Hes 7 result in severe somite fusion, indicating that Hes 7 constitutes an essential component of the segmentation clock. Interestingly, expression of the related gene Hes 1 also oscillates with a periodicity of about 2 hours in many cell types. Both sustained Hes 1 expression and loss of Hes 1 activity lead to retardation of the $\mathrm{G}_{1}$ phase of the cell cycle, suggesting that Hes 1 oscillation with an ultradian rhythm is required for efficient cell proliferation. Both Hes 1 and Hes 7 oscillations are regulated by negative feedback and rapid degradation of their gene products. Strikingly, expression of other factors such as Stat-Socs and Smad signaling molecules also display ultradian rhythms. All of these data suggest that ultradian oscillations are more general responses than were previously thought and that oscillatory and sustained gene expression results in different biological outcomes.
\end{abstract}

\section{INTRODUCTION}

It is well known that many day and night activities have a circadian rhythm, which has a periodicity of about 24 hours. Some biological activities, however, are known to have rhythms with shorter periodicities called ultradian rhythms. For example, secretion of hormones and changes of plasma glucose levels are pulsatile with periodicities of a few hours (Tannenbaum and Martin 1976; Simon et al. 1987). Another example is somite formation, which occurs every 2 hours in the mouse and every several hours in humans. Recently, characterization of the molecular mechanism of periodic somite formation has been greatly advanced.

Somites are transient metameric structures located at either side of the neural tube, and they later give rise to the vertebral column, ribs, skeletal muscles, and subcutaneous tissues. Somites are formed by segmentation of the anterior ends of the presomitic mesoderm (PSM) (Fig. 1a). In mouse embryos, a bilateral pair of somites are formed every 2 hours, suggesting that this process is controlled by a biological clock with a 2-hour periodicity, and it has been called the segmentation clock (Bessho and Kageyama 2003; Pourquié 2003; Aulehla and Herrmann 2004; Gridley 2006). During this process, the basic helixloop-helix (bHLH) genes Hes 1 and Hes 7 display oscillatory expression in PSM cells, leading to generation of a pair of somites after each cycle (Bessho et al. 2001a). In particular, Hes 7 is an essential component of the segmentation clock. In the absence of Hes 7 , somites become severely fused (Bessho et al. 2001b). Interestingly, Hes 1 also shows oscillatory expression in many cell types, in addition to PSM cells, suggesting that oscillation is not unique to PSM cells and that Hes 1 oscillation may regulate the timing of many biological events (Jouve et al. 2000; Hirata et al. 2002). It has been shown that other genes are also cyclically expressed by many cell types, raising the possibility that oscillatory expression is more general than was previously thought. In this chapter, we

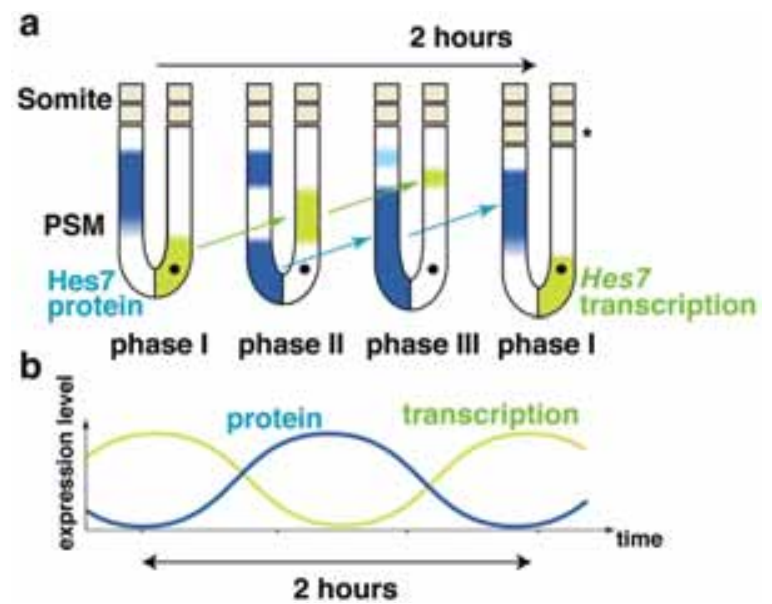

Figure 1. Hes7 oscillation in the somite segmentation. (a) In mouse embryos, a bilateral pair of somites are formed every 2 hours by segmentation of the anterior ends of the PSM (asterisk). Expression of the Hes 7 intron (corresponding to the region where the Hes 7 gene is transcribed, green) is initiated in the posterior PSM (phase I), and this expression region is then propagated into the anterior PSM (phase II), finally reaching S-1 (phase III). By the time $\mathrm{S}-1$ becomes S0, Hes 7 intron expression disappears, and the next expression occurs again in the posterior PSM (i.e., it returns to phase I). The Hes 7 transcription region (green) and the Hes 7-protein-expressing region (blue) are mutually exclusive in all three phases, suggesting that Hes 7 transcription occurs only when Hes 7 protein is not expressed. $\mathrm{S} 0$ is the next somitic region formed, whereas S-1 is the region that becomes a somite following S0. Anterior is top. (b) Dynamic change in Hes 7 expression is the result of oscillatory expression in individual PSM cells (indicated by a dot in $a$ ). (Adapted, with permission, from Kageyama et al. 2007a [C John Wiley \& Sons].) 
discuss the mechanism and the significance of Hes $1 / \mathrm{Hes} 7$ oscillations and other ultradian oscillators in biological events.

\section{THE ROLES AND THE MECHANISM OF HES7 OSCILLATION IN THE SEGMENTATION CLOCK}

Hes7 expression dynamically changes in PSM and has been classified as occuring in three phases (Fig. 1a). Expression of the Hes 7 intron (corresponding to the region where $\mathrm{Hes} 7$ gene is transcribed) is initiated in the posterior PSM (phase I), and this region is then propagated into the anterior PSM (phase II), finally reaching S1 (phase III) (Fig. 1a, green). By the time S-1 becomes S0 (the next somitic region formed), Hes 7 intron expression disappears, whereas the next expression of Hes 7 intron occurs again in the posterior PSM (i.e., it returns to phase I). This dynamic change in gene expression is the result of oscillatory expression of Hes 7 in individual PSM cells (Fig. 1b). Cells located laterally display oscillation inphase, but this is delayed in anterior cells compared to posterior cells. As a result, the expression domain moves from the posterior to the anterior region.

Interestingly, the Hes 7 transcription region (Fig. 1a, green) and the Hes7 protein-expressing region (Fig. 1a, blue) are mutually exclusive in all three phases, suggesting that Hes 7 transcription occurs only when Hes7 protein is not expressed (Bessho et al. 2001a, 2003). In agreement with this notion, the Hes7 protein can repress Hes 7 transcription by directly binding to its own promoter (Bessho et al. 2003). Furthermore, in the absence of functional Hes 7 protein, the Hes 7 gene is constitutively transcribed in the PSM, whereas Hes 7 transcription is constitutively repressed when Hes7 protein is stabilized (Bessho et al. 2003). These data together suggest that Hes 7 oscillation is regulated by negative autoregulation as follows (Fig. 2). In the PSM, Notch signaling induces expression of Hes7, which represses its own expression by binding to its own promoter. When the promoter is repressed, both $\mathrm{Hes} 7$

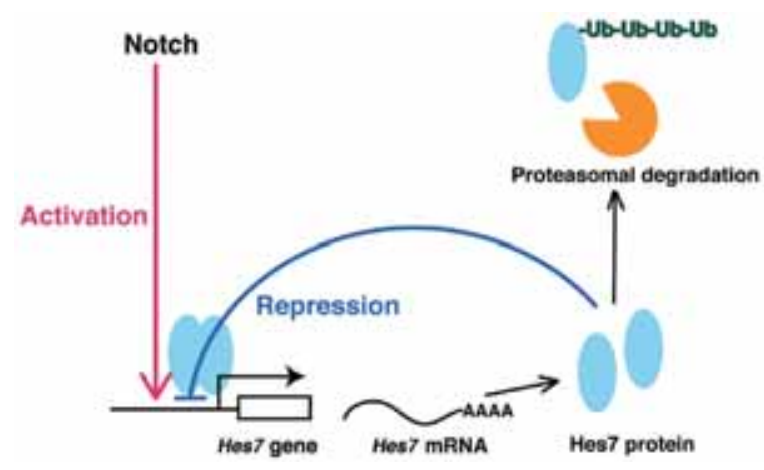

Figure 2. Hes7 oscillation regulated by negative autoregulation. Notch signaling induces expression of Hes7, which represses its own expression by binding to its own promoter. When the promoter is repressed, both Hes7 mRNA and protein disappear rapidly because they are extremely unstable, allowing the next round of expression. In this way, Hes 7 autonomously starts oscillatory expression.
mRNA and protein disappear rapidly because they are extremely unstable, allowing the next round of expression.

Hes 1 displays an expression pattern similar to that of Hes 7 in the PSM. Hes1, a transcriptional repressor, can repress its own expression by directly binding to the promoter (Sasai et al. 1992; Takebayashi et al. 1994), and Hes 1 oscillation is also regulated by this negative autoregulation (Hirata et al. 2002), although the expression is maintained in $\mathrm{S} 0$ and in the somitic regions, unlike Hes 7.

It has been shown that expression of Lunatic fringe (Lfng), a glycosyltransferase gene that inhibits Notch activity (Brückner et al. 2000; Moloney et al. 2000), oscillates in phase with Hes 7 (Bessho et al. 2001b). In Lfngnull mice, Hes 7 expression still oscillates, although the expression is somewhat affected (Niwa et al. 2007), suggesting that Hes 7 oscillation does not depend on Lfng oscillation. In contrast, in Hes 7-null mice, Lfng is constitutively expressed throughout the PSM (Bessho et al. 2001b). Conversely, stabilized Hes7 constitutively represses $L f n g$ expression (Bessho et al. 2003). These data indicate that $L f n g$ oscillation depends on periodic repression by Hes 7 .

Both Hes7 and Lfing have an essential role in somite segmentation. In Hes 7-null and Lfng-null mice, somites severely fuse (Evrard et al. 1998; Zhang and Gridley 1998; Bessho et al. 2001b). Interestingly, transgenic mice that constitutively express Hes 7 or Lfng throughout the PSM also display severe somite fusion (Serth et al. 2003; Hirata et al. 2004). Thus, both sustained expression and loss of expression of Hes 7 or Lfng disturb somite segmentation, suggesting that oscillatory expression of both genes is required for this process.

\section{MATHEMATICAL SIMULATION OF HES7 OSCILLATION}

Hes1/Hes 7 oscillations can be mathematically simulated, based on the negative autoregulation mechanism described above (Jensen et al. 2003; Lewis 2003; Monk 2003; Hirata et al. 2004). The simulation for Hes7 oscillation consists of two differential equations (Hirata et al. 2004).

$$
\begin{aligned}
\frac{d p(t)}{d t} & =a m\left(t-T_{p}\right)-b p(t) \\
\frac{d m(t)}{d t} & =f\left(p\left(t-T_{m}\right)\right)-c m(t) \\
f(p) & =\frac{k}{1+\left(\frac{p}{p_{c r i t}}\right)^{2}}
\end{aligned}
$$

where $p(t)$ and $m(t)$ are the quantities of functional Hes7 protein and mRNA, respectively, per cell at time $t$, and $f(p)$ is the rate of initiation of transcription, which is negatively regulated by a dimer of the protein $p$ present at the time of initiation. $a$ is the rate constant for translation, whereas $b$ and $c$ are the degradation rate constants for protein and mRNA, respectively.

According to this simulation, the instability of Hes7 protein is very important for sustained oscillation. The 


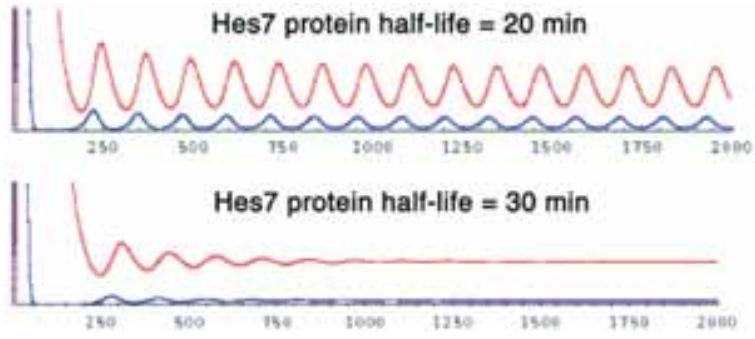

Figure 3. Stabilization of Hes7 protein leads to damped oscillation. The half-life of Hes7 protein is about 20 minutes, and this value is important for sustained oscillations. If the half-life becomes 30 minutes, Hes7 oscillations become damped after three to four cycles. (Adapted, with permission, from Hirata et al. 2004 [Nature Publishing Group].)

half-life of Hes 7 protein is about 20 minutes, but if it reaches 30 minutes instead of 20 minutes, the simulation predicts that Hes 7 oscillation becomes damped after several cycles (Fig. 3). However, this prediction is counterintuitive, which suggests that even if the half-life is 30 minutes, Hes 7 oscillations could continue in a sustained manner, although the period would be a little longer than 2 hours. The prediction of the mathematical simulation was experimentally evaluated by making mutant mice that express stabilized Hes 7 protein. Hes7 protein with a K14R mutation (the 14th amino acid residue [lysine] of Hes 7 protein is mutated to arginine) has a normal repressor activity, but its half-life is about 30 minutes. A K14R point mutation was introduced into the Hes7 locus of ES cells, and from these ES cells, the mutant mice that express Hes 7 with the K14R mutation were generated. Interestingly, although three or four pairs of somites are segmented, the subsequent somites fuse severely in these mutant mice (Hirata et al. 2004). Furthermore, Hes7 oscillation becomes damped after three to four cycles (Hirata et al. 2004). These data agree well with the prediction of the mathematical simulation and indicate that intuition alone is insufficient and that mathematical modeling is required to understand the dynamics of Hes oscillations.

\section{REAL-TIME MONITORING OF HES OSCILLATIONS}

To further examine Hes oscillation patterns, we established a real-time imaging system for $\mathrm{Hes} 1 / \mathrm{Hes} 7$ expression, where the Hes $1 /$ Hes 7 promoter-driven ubiquitinated luciferase is used as a reporter (Masamizu et al. 2006; and our unpublished data). Because the normal luciferase protein has a half-life of several hours, it is too stable to monitor 2-hour cycle oscillations. Such a reporter protein would be accumulated after several cycles of oscillations. In contrast, the ubiquitinated luciferase has a half-life of about 10 minutes and can be used for monitoring oscillations with a 2-hour periodicity. The system with the ubiquitinated luciferase reporter successfully enabled observation of Hes1/Hes7 oscillations in real time in PSM, offering a powerful tool to understand the dynamics of Hes1/Hes 7 oscillations. On the basis of real-time imaging, a spatiotemporal profile was reproduced (Fig. 4a) (Kageyama et al. 2007a). This profile suggests that each individual PSM cell seems to experience five cycles of Hes1 oscillations before entering the S0 region (the next somite region) about embryonic day 10.5. Interestingly, the period of the first three cycles is about 2 hours, whereas that of the fourth and fifth cycles is longer than 2 hours (Fig. 4b). Cells experiencing the first three cycles are located in the posterior PSM, whereas those experiencing the last two cycles are located in the anterior PSM, indicating that the period of Hes1 oscillations is different between the posterior and the anterior PSM. The different periods between the posterior and the anterior PSM could be regulated by Fgf signaling (Niwa et al. 2007), which forms a posterior-toanterior gradient in the PSM, although the precise mechanism remains to be determined.

Hes 1 expression oscillates in a stable manner in PSM cells but not in dissociated PSM cells. In these cells, the periods of Hes 1 oscillations are variable from cycle to cycle, indicating that Hes1 oscillation in individual PSM cells is unstable (Masamizu et al. 2006). Thus, negative autoregulation can create only an unstable oscillator, suggesting that cell-cell communication and/or extracellular signals are important for stable Hes1 oscillations. We observed the same features in Hes7 oscillations (our unpublished data). It is likely that Notch signaling is involved in cell-cell communication (Jiang et al. 2000; Horikawa et al. 2006), although further analysis is required to clarify this issue.

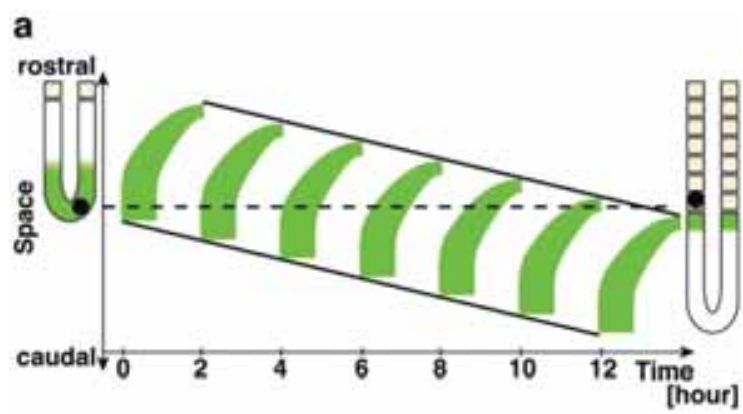

b

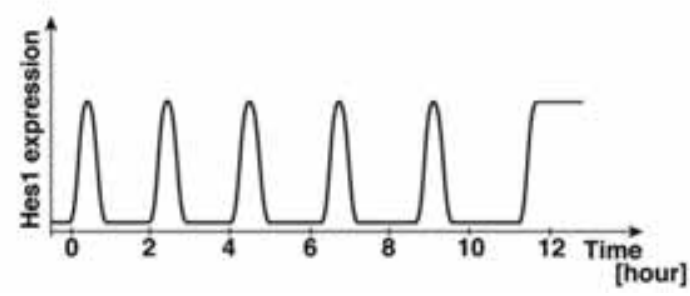

Figure 4. Spatiotemporal profile of Hes1 oscillation in PSM. (a) A spatiotemporal profile of Hes1 oscillation in PSM was reproduced, based on the real-time imaging analysis (Masamizu et al. 2006; Kageyama et al. 2007a). (b) Hes1 expression in individual PSM cells. Each PSM cell seems to experience five cycles of Hes 1 oscillation after getting out of the tail bud and before reaching the $\mathrm{S} 0$ region. The periodicity of the first three cycles is about 2 hours, whereas that of the last two cycles is longer, suggesting that Hes 1 oscillation is differentially regulated between the anterior and the posterior PSM. (Adapted, with permission, from Kageyama et al. 2007a [@ John Wiley \& Sons].) 


\section{HES1 OSCILLATION IN NON-PSM CELLS}

Interestingly, oscillatory expression is not unique to PSM cells. Hes1 is widely expressed by many cell types such as fibroblasts and neuroblasts (Kageyama et al. 2007b). After serum stimulation or Notch activation, Hes 1 expression oscillates with a periodicity of about 2 hours in many cell types, suggesting that Hes1 may regulate many biological processes as a biological clock with a 2-hour cycle (Hirata et al. 2002). Both sustained Hes1 expression and knock down of Hes1 activity reduce proliferation of fibroblasts, suggesting that oscillatory Hes 1 expression is required for efficient proliferation of fibroblasts (Yoshiura et al. 2007). Furthermore, persistent Hes1 expression also inhibits proliferation and differentiation of neuroblasts (Baek et al. 2006). It seems that Hes1 promotes certain steps but inhibits other steps of the cell cycle, although it remains to be determined at which steps of the cell cycle Hes1 regulation actually occurs.

Northern and western blot analyses indicate that in nonPSM cells, Hes1 oscillations seem to be damped after three to six cycles (Hirata et al. 2002). Real-time monitoring at the single-cell level revealed that Hes1 oscillation continues in each individual cell even after 2 days, but because the period of each cycle is variable from cycle to cycle and from cell to cell, Hes 1 oscillation among cells soon goes out of synchrony (Masamizu et al. 2006). Thus, damping of Hes1 oscillation in the population is not due to damped oscillation in all individual cells, but it is due to desynchronization between cycling cells. Interestingly, this unstable period of Hes1 oscillation of fibroblasts is very similar to that of dissociated PSM cells.

\section{OTHER ULTRADIAN OSCILLATORS}

In addition to Hes1/Hes7, many other factors have been shown to display oscillatory expression in PSM cells (Dequéant et al. 2006). Similarly, several other factors have been shown to display oscillatory expression in nonPSM cells. NF- $\kappa \mathrm{B}$ is trapped in the cytoplasm by $\mathrm{I} \kappa \mathrm{B}$, but in the presence of inducing signals such as tumor necrosis factor- $\alpha$ (TNF- $\alpha$, ) I $\kappa$ B is degraded, and NF- $\kappa$ B is released and transferred into the nucleus, where it induces expression of many genes. One of them is $I \kappa \mathrm{B}$, which again traps NF- $\kappa B$. However, if the inducing signals are still present, I $\mathrm{\kappa B}$ is degraded and NF- $\kappa \mathrm{B}$ is translocated to the nucleus again. As a result, the localization of NF$\kappa \mathrm{B}$ oscillates between the nucleus and cytoplasm $(\mathrm{N}-\mathrm{C}$ oscillation) (Hoffmann et al. 2002; Nelson et al. 2004). It has been shown that $\mathrm{p} 53$ expression also oscillates due to the negative feedback of Mdm2 (Bar-Or et al. 2000; Lahav et al. 2004). These observations suggest the possibility that other molecules forming negative feedback loops can also display oscillatory responses.

To identify new ultradian oscillators, RNA was prepared from fibroblasts every 30 minutes after serum stimulation and subjected to microarray analyses, which revealed oscillatory responses of Socs3 and Smad6 (Yoshiura et al. 2007). Expression of both genes oscillates with a period of about 2 hours, although the peaks appear at different times. Socs 3 is the downstream target of the
Jak-Stat signaling (Starr et al. 1997; Levy and Darnell 2002; Yu and Jove 2004). Jak2 activates Stat3 by phosphorylation, and phosphorylated Stat3 (p-Stat3) forms a dimer, enters the nucleus, and induces expression of many genes (Fig. 5). One of them is Socs3, which antagonizes Jak-dependent activation of Stat3, thus forming a negative feedback loop. Because Socs3 expression oscillates, Jak-dependent activation of Stat 3 should be periodically inhibited. In agreement with this notion, formation of $\mathrm{p}$ Stat3 is also found to be oscillatory. Thus, the Jak-Stat3Socs 3 negative feedback loop generates p-Stat3/Socs3 oscillations (Fig. 5) (Yoshiura et al. 2007). Importantly, inhibition of p-Stat3/Socs3 oscillations abolishes Hes 1 oscillation, suggesting that Hes1 oscillation is not solely regulated by negative feedback. p-Stat 3 seems to periodically destabilize Hes 1 protein, and this periodic destabilization may be important for Hes 1 oscillation (Yoshiura et al. 2007). Conversely, it was previously shown that Hes 1 promotes Jak-dependent activation of Stat3 by physical interaction (Kamakura et al. 2004). Thus, it is likely that Hes 1 oscillation and p-Stat3/Socs3 oscillations are coupled with and depend on each other.

Smad6 is the downstream target of Smad1 signaling (Imamura et al. 1997; Massagué and Wotton 2000; ten Dijke et al. 2000). Signaling molecules such as bone morphogenetic protein (BMP) activate Smad1/5/8 by phosphorylation. Phosphorylated Smad1/5/8 (p-Smad1/5/8) up-regulates expression of downstream genes including Smad6, which then inhibits $p-S m a d 1 / 5 / 8$ formation, constituting a negative feedback loop (Fig. 6). Because Smad6 expression oscillates, Smad1/5/8 activation should be periodically inhibited. In agreement with this notion, formation of $\mathrm{p}-\mathrm{Smad} 1$ is also found to be oscillatory. Thus, the Smad1-Smad6 negative feedback loop generates p-Smad1/Smad6 oscillations (Fig. 6) (Yoshiura et al. 2007). However, unlike p-Stat3/Socs 3 oscillations, p-Smad1/Smad6 oscillations are not involved in Hes 1 oscillation, suggesting that the p-Smad1/Smad6 oscillations function independently of Hes 1 oscillation. In

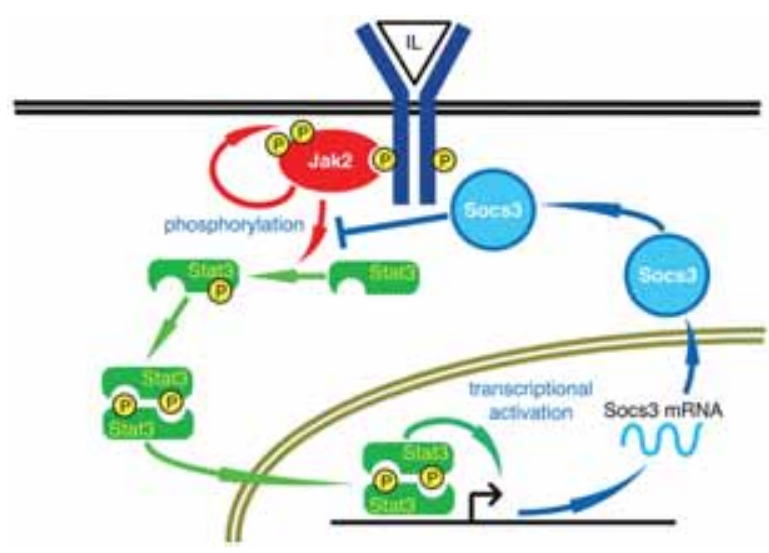

Figure 5. p-Stat3/Socs 3 oscillations. Jak 2 activates Stat 3 by phosphorylation. Phosphorylated Stat3 (p-Stat3) enters the nucleus and induces expression of downstream genes. One of them is Socs3, which antagonizes formation of $\mathrm{p}$-Stat3. This negative feedback loop generates oscillatory expression of p-Stat3 and Socs3. 


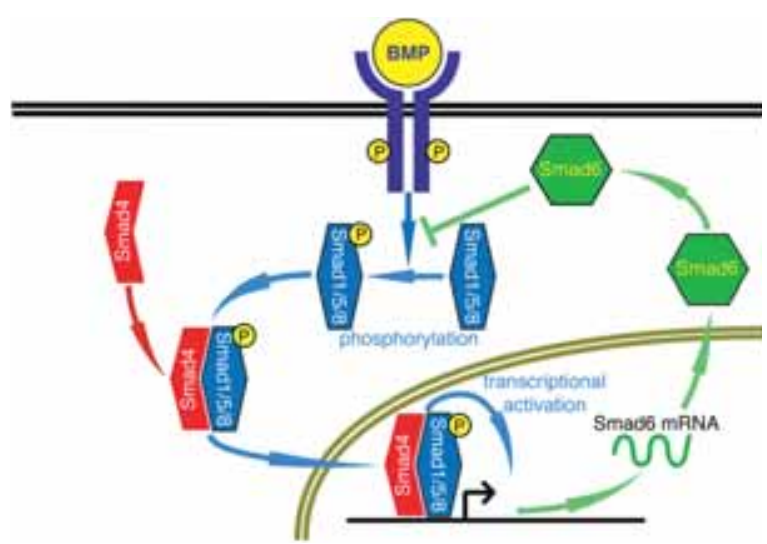

Figure 6. p-Smad1/Smad6 oscillations. Signaling molecules such as BMP activate Smad1/5/8 by phosphorylation. Phosphorylated Smad1/5/8 (p-Smad1/5/8) up-regulates expression of downstream genes including Smad6, which then inhibits $p$-Smad1/5/8 formation, constituting a negative feedback loop. Because Smad6 expression oscillates, Smad1/5/8 activation should be periodically inhibited. In agreement with this notion, formation of $\mathrm{p}-\mathrm{Smad} 1$ is found to be oscillatory. The Smad1/5/8-Smad6 negative feedback loop generates $\mathrm{p}$-Smad1/5/8 and Smad6 oscillations.

agreement with this notion, Hes 1 and Socs 3 oscillate in phase, whereas Hes1 and Smad6 do not.

\section{POSSIBLE SIGNIFICANCE OF ULTRADIAN OSCILLATIONS}

Although Hes1 oscillation is required for efficient cell proliferation, the expression modes of the downstream target genes are not well-characterized. In our microarray analyses, we did not identify oscillatory downstream target genes for Stat, Smad, and Hes oscillators. Expression of some downstream genes could oscillate (Fig. 7a), but synchrony of oscillation among cells might easily be lost. In this case, real-time monitoring of gene expression at the single-cell level is required. Hes 1 oscillations would produce different conditions in different cells, leading to different responses. For example, when differentiation is induced, cells in a low Hes1 phase, but not those in a high Hes1 phase, would respond to the induction. Thus, Hes1 oscillations could make responsive and unresponsive cells to the same signals.

If the downstream target gene products are relatively stable, they do not respond in an oscillatory manner. For example, expression of such downstream factors can be maintained within a certain range by periodic down-regulation by Hes 1 oscillation (Fig. 7b). If products of downstream target genes are even more stable, transcriptional induction by activators and periodic repression by Hes 1 oscillation could lead to up-regulation of downstream target factors in a stepwise manner (Fig. 7c). In this case, if the expression level reaches a certain value, the next event could happen. Thus, the information about the number of cycles can be converted into the timing of the next event. Further analysis of downstream target genes will be required to reveal more precise significance and roles of ultradian oscillators.
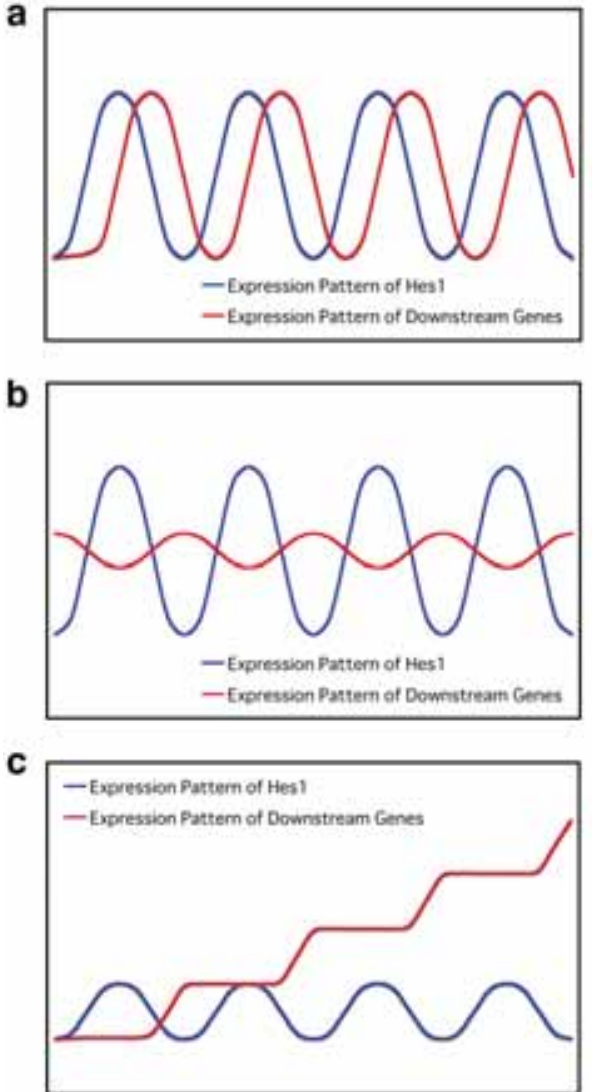

Figure 7. Downstream events of ultradian oscillators. Downstream factors for Hes1/Hes7 oscillations could display at least three different expression patterns. (a) If products of downstream genes are unstable, their expression could oscillate like Hes 1 and Hes7. (b) If downstream factors are relatively stable. their expression can be maintained within a certain range by periodic down-regulation by Hes $1 / \mathrm{Hes} 7$ oscillations. (c) If downstream factors are very stable, transcriptional induction by activators and periodic repression by Hes1/Hes7 oscillations could lead to up-regulation of downstream target factors in a stepwise manner. If the expression level reaches a certain value, the next event could happen. In this case, the information about the number of cycles can be converted into the timing of the next event.

\section{CONCLUSIONS}

It has been shown that many genes display expression with ultradian rhythms. In the case of Hes1, although the expression seems to be stationary on northern and western analyses without serum stimulation, it is actually oscillatory at the single-cell level. Oscillatory expression of Hes 1 is required for efficient cell proliferation, as sustained expression retards the $\mathrm{G}_{1}$ phase of the cell cycle. Similarly, oscillatory expression of Hes7 is required for somite segmentation, as sustained expression leads to severe somite fusion. Thus, oscillatory and sustained gene expression result in different biological outcomes. Ultradian oscillations are regulated by negative feedback, but it seems that the negative feedback loop alone generates only unstable oscillators. Cell-cell communication or other extracellular signals are required for stable oscillators. Reconstitution of such oscillators in non-PSM cells 
would be required to understand the basic mechanism of stable oscillations.

Other molecules forming negative feedback loops are also shown to display ultradian rhythms, suggesting that ultradian oscillations are more general responses than were previously thought. Further characterization of ultradian oscillators and their downstream targets will give us more insight into the understanding of how cells measure time in many biological processes.

\section{ACKNOWLEDGMENTS}

This work was supported by the grants-in-aid from the Genome Network Project and the Ministry of Education, Culture, Sports, Science and Technology of Japan. S.Y., Y.M., and Y.N. were supported by the 21 st Century COE Program of the Ministry of Education, Culture, Sports, Science and Technology of Japan and by research fellowships of the Japan Society for the Promotion of Science for Young Scientists.

\section{REFERENCES}

Aulehla A. and Herrmann B.G. 2004. Segmentation in vertebrates: Clock and gradient finally joined. Genes Dev. 18: 2060.

Baek J.H., Hatakeyama J., Sakamoto S., Ohtsuka T., and Kageyama R. 2006. Persistent and high levels of Hes1 expression regulate boundary formation in the developing central nervous system. Development 133: 2467

Bar-Or R.L., Maya R., Segel L.A., Alon U., Levine A.J., and Oren M. 2000. Generation of oscillations by the p53-Mdm2 feedback loop: A theoretical and experimental study. Proc. Natl. Acad. Sci. 97: 11250.

Bessho Y. and Kageyama R. 2003. Oscillations, clocks and segmentation. Curr. Opin. Genet. Dev. 13: 379.

Bessho Y., Hirata H., Masamizu Y., and Kageyama R. 2003. Periodic repression by the bHLH factor Hes7 is an essential mechanism for the somite segmentation clock. Genes Dev. 17: 1451

Bessho Y., Miyoshi G., Sakata R., and Kageyama R. 2001a. Hes 7: A bHLH-type repressor gene regulated by Notch and expressed in the presomitic mesoderm. Genes Cells 6: 175.

Bessho Y., Sakata R., Komatsu S., Shiota K., Yamada S., and Kageyama R. 2001b. Dynamic expression and essential functions of Hes 7 in somite segmentation. Genes Dev. 15: 2642.

Brückner K., Perez L., Clausen H., and Cohen S. 2000. Glycosyltransferase activity of Fringe modulates Notch-Delta interactions. Nature 406: 411.

Dequéant M.-L. Glynn E., Gaudenz K., Wahl M., Chen J., Mushegian A., and Pourquié O. 2006. A complex oscillating network of signaling genes underlies the mouse segmentation clock. Science 314: 1595.

Evrard Y.A., Lun Y., Aulehla A., Gan L., and Johnson R.L. 1998. lunatic fringe is an essential mediator of somite segmentation and patterning. Nature 394: 377.

Gridley T. 2006. The long and short of it: Somite formation in mice. Dev. Dyn. 235: 2330.

Hirata H., Bessho Y., Kokubu H., Masamizu Y., Yamada S., Lewis J., and Kageyama R. 2004. Instability of Hes7 protein is crucial for the somite segmentation clock. Nat. Genet. 36: 750.

Hirata H., Yoshiura S., Ohtsuka T., Bessho Y., Harada T., Yoshikawa K., and Kageyama R. 2002. Oscillatory expression of the bHLH factor Hes1 regulated by a negative feedback loop. Science 298: 840 .

Hoffmann A., Levchenko A., Scott M.L., and Baltimore D.

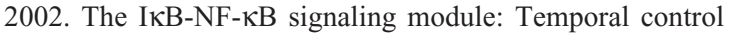
and selective gene activation. Science 298: 1241.
Horikawa K., Ishimatsu K., Yoshimoto E., Kondo S., and Takeda H. 2006. Noise-resistant and synchronized oscillation of the segmentation clock. Nature 441: 719.

Imamura T., Takase M., Nishihara A., Oeda E., Hanai J., Kawabata M., and Miyazono K. 1997. Smad6 inhibits signalling by the TGF- $\beta$ superfamily. Nature 389: 622 .

Jensen M.H., Sneppen K., and Tiana G. 2003. Sustained oscillations and time delays in gene expression of protein Hes1. FEBS Lett. 541: 176.

Jiang Y.J., Aerne B.L., Smithers L., Haddon C., Ish-Horowicz D., and Lewis J. 2000. Notch signalling and the synchronization of the somite segmentation clock. Nature 408: 475.

Jouve C., Palmeirim I., Henrique D., Beckers J., Gossler A., IshHorowicz D., and Pourquié O. 2000. Notch signalling is required for cyclic expression of the hairy-like gene HES1 in the presomitic mesoderm. Development 127: 1421.

Kamakura S., Oishi K., Yoshimatsu T., Nakafuku M., Masuyama N., and Gotoh Y. 2004. Hes binding to STAT3 mediates crosstalk between Notch and JAK-STAT signaling. Nat. Cell Biol. 6: 547.

Kageyama R., Masamizu Y., and Niwa Y. 2007a. Oscillator mechanism of Notch pathway in the segmentation clock. Dev. Dyn. 236: 1403.

Kageyama R., Ohtsuka T., and Kobayashi T. 2007b. The Hes gene family: Repressors and oscillators that orchestrate embryogenesis. Development 134: 1243.

Lahav G., Rosenfeld N., Sigal A., Geva-Zatorsky N., Levine A.J., Elowitz M.B., and Alon U. 2004. Dynamics of the p53Mdm2 feedback loop in individual cells. Nat. Genet. 36: 147.

Levy D.E. and Darnell J.E., Jr. 2002. Stats: Transcriptional control and biological impact. Nat. Rev. Mol. Cell Biol. 3: 651.

Lewis J. 2003. Autoinhibition with transcriptional delay: A simple mechanism for the zebrafish somitogenesis oscillator. Curr. Biol. 13: 1398.

Masamizu Y., Ohtsuka T., Takashima Y., Nagahara H., Takenaka Y., Yoshikawa K., Okamura H., and Kageyama R. 2006. Real-time imaging of the somite segmentation clock: Revelation of unstable oscillators in the individual presomitic mesoderm cells. Proc. Natl. Acad. Sci. 103: 1313.

Massagué J. and Wotton D. 2000. Transcriptional control by the TGF- $\beta /$ Smad signaling system. EMBO J. 19: 1745.

Moloney D.J., Panin V.M., Johnston S.H., Chen J., Shao L., Wilson R., Wang Y., Stanley P., Irvine K.D., Haltiwanger R.S., and Vogt T.F. 2000. Fringe is a glycosyltransferase that modifies Notch. Nature 406: 369.

Monk N.A. 2003. Oscillatory expression of Hes1, p53, and NF$\mathrm{\kappa B}$ driven by transcriptional time delays. Curr. Biol. 13: 1409.

Nelson D.E., Ihekwaba A.E., Elliott M., Johnson J.R., Gibney C.A., Foreman B.E., Nelson G., See V., Horton C.A., Spiller D.G., Edwards S.W., McDowell H.P., Unitt J.F., Sullivan E., Grimley R., Benson N., Broomhead D., Kell D.B., and White M.R. 2004. Oscillations in NF- $\mathrm{KB}$ signaling control the dynamics of gene expression. Science 306: 704.

Niwa Y., Masamizu Y., Liu T., Nakayama R., Deng C.-X., and Kageyama R. 2007. The initiation and propagation of Hes7 oscillation are cooperatively regulated by Fgf and Notch signaling in the somite segmentation clock. Dev. Cell 13: 298.

Pourquié O. 2003. The segmentation clock: Converting embryonic time into spatial pattern. Science 301: 328 .

Sasai Y., Kageyama R., Tagawa Y., Shigemoto R., and Nakanishi S. 1992. Two mammalian helix-loop-helix factors structurally related to Drosophila hairy and Enhancer of split. Genes Dev. 6: 2620.

Serth K., Schuster-Gossler K., Cordes R., and Gossler A. 2003. Transcriptional oscillation of Lunatic fringe is essential for somitogenesis. Genes Dev. 17: 912.

Simon C., Follenius M., and Brandenberger G. 1987. Postprandial oscillations of plasma glucose, insulin and Cpeptide in man. Diabetologia 30: 769 .

Starr R., Willson T.A., Viney E.M., Murray L.J., Rayner J.R., Jenkins B.J., Gonda T.J., Alexander W.S., Metcalf D., Nicola N.A., and Hilton D.J. 1997. A family of cytokine-inducible inhibitors of signalling. Nature 387: 917.

Takebayashi K., Sasai Y., Sakai Y., Watanabe T., Nakanishi S., 


\section{ULTRADIAN OSCILLATIONS}

and Kageyama R. 1994. Structure, chromosomal locus, and promoter analysis of the gene encoding the mouse helix-loophelix factor HES-1: Negative autoregulation through the multiple $\mathrm{N}$ box elements. J. Biol. Chem. 269: 5150.

Tannenbaum G.S. and Martin J.B. 1976. Evidence for an endogenous ultradian rhythm governing growth hormone secretion in the rat. Endocrinology 98: 562.

ten Dijke P., Miyazono K., and Heldin C.-H. 2000. Signaling inputs converge on nuclear effectors in TGF- $\beta$ signaling.
Trends Biosci. 25: 64 .

Yoshiura S., Ohtsuka T., Takenaka Y., Nagahara H., Yoshikawa K., and Kageyama R. 2007. Ultradian oscillations of Stat, Smad and Hes1 expression in response to serum. Proc. Natl. Acad. Sci. 104: 11292.

Yu H. and Jove R. 2004. The STATS of cancer: New molecular targets come of age. Nat. Rev. Cancer 4: 97.

Zhang N. and Gridley T. 1998. Defects in somite formation in lunatic fringe-deficient mice. Nature 394: 374 


\section{$\overbrace{\mathrm{CSH}}^{\infty}$ Cold Spring Harbor Symposia SYMPOSIA}

\section{Ultradian Oscillators in Somite Segmentation and Other Biological Events}

R. Kageyama, S. Yoshiura, Y. Masamizu, et al.

Cold Spring Harb Symp Quant Biol 2007 72: 451-457

Access the most recent version at doi:10.1101/sqb.2007.72.012

References This article cites 42 articles, 18 of which can be accessed free at: http://symposium.cshlp.org/content/72/451.full.html\#ref-list-1

License

Email Alerting Receive free email alerts when new articles cite this article - sign up in the box at the Service top right corner of the article or click here. 